\title{
Understanding Wear Induced Sub-surface Deformation Using FIB and EBSD
}

\author{
J.R. Michael and S. V. Prasad \\ Sandia National Laboratories, PO Box 5800, Albuquerque, NM 87185-0886
}

Plastic strains during sliding contact result in sub-surface layers whose microstructures are different from the bulk. [1] Microstructural evolution underneath wear surfaces of metallic materials has been subject of numerous studies. Previous studies relied on conventional TEM specimen preparation techniques to study wear-induced deformation under heavy loads. [2] The use of metallic MEMS (microelectromechanical systems) requires that friction and wear behavior in low load regimes be better understood in order to ascertain device reliability. In this study we applied FIB techniques to prepare samples at specific locations on a wear scar and visualize the deformation induced microstructural changes by EBSD.

A custom made friction and wear tester was utilized to produce wear scars on (111), (110) and (001) Ni single crystals in specific crystallographic directions using a $3.125 \mathrm{~mm}$ diameter silicon nitride ball as the counterface. A normal load of $100 \mathrm{mN}$ or $1 \mathrm{~N}$ was applied on the ball by deadweights. A transducer in the load arm measures the friction force. A rotary specimen stage was built to align the single crystals in specific crystallographic directions marked by EBSD and FIB. Friction and wear tests were run in unidirectional mode for 1000 cycles. Thin TEM cross sections were prepared parallel to the wear track using FIB. The FIB samples were transferred to carbon coated TEM supports. EBSD orientation mapping was performed on the FIB prepared samples to allow the deformation to be visualized through orientation changes.

Fig 1a and $\mathrm{b}$ are ion induced secondary electron images of the FIB prepared samples from wear scars generated by $100 \mathrm{mN}$ and $1 \mathrm{~N}$ loads for 1000 cycles on (111) Ni single crystals in the $<110>$ direction. The top of each image is the FIB deposited Pt protective layer. Figure $2 \mathrm{a}$ and $\mathrm{b}$ are EBSD orientation maps (see inset legend) with respect to the wear direction (horizontal) for the same samples as shown in Figure 1. The large amount of deformation is clearly visible in the orientation maps. Grain rotations of as large as $60^{\circ}$ have been measured after wear deformation. Monitoring of the friction force during wear testing of this orientation and others demonstrates that there is a linkage between the friction force and the amount of deformation that occurs.

Combined FIB sectioning and TEM and EBSD analysis have provided new insights into the microstructural changes that are associated with friction and wear of surfaces. Large grain rotations have been observed under wear scars even at the low normal forces used in this study. Eventually the deformation causes the $\mathrm{Ni}$ single crystal to break down into a fine grained region just below the wear surface. This work has found that friction forces are related to the degree of deformation that occurs and that this varies with surface plane and wear direction.

References

[1] D. A. Rigney et al., Wear, 53 (1979) 345.

[2] P. Heilman et al., Acta Metall.. 31 (1983) 1293.

[3] Sandia is a multiprogram laboratory operated by Sandia Corporation, a Lockheed Martin Company, for the United Stated Department of Energy (DOE) under contract DE-AC0494AL85000. 

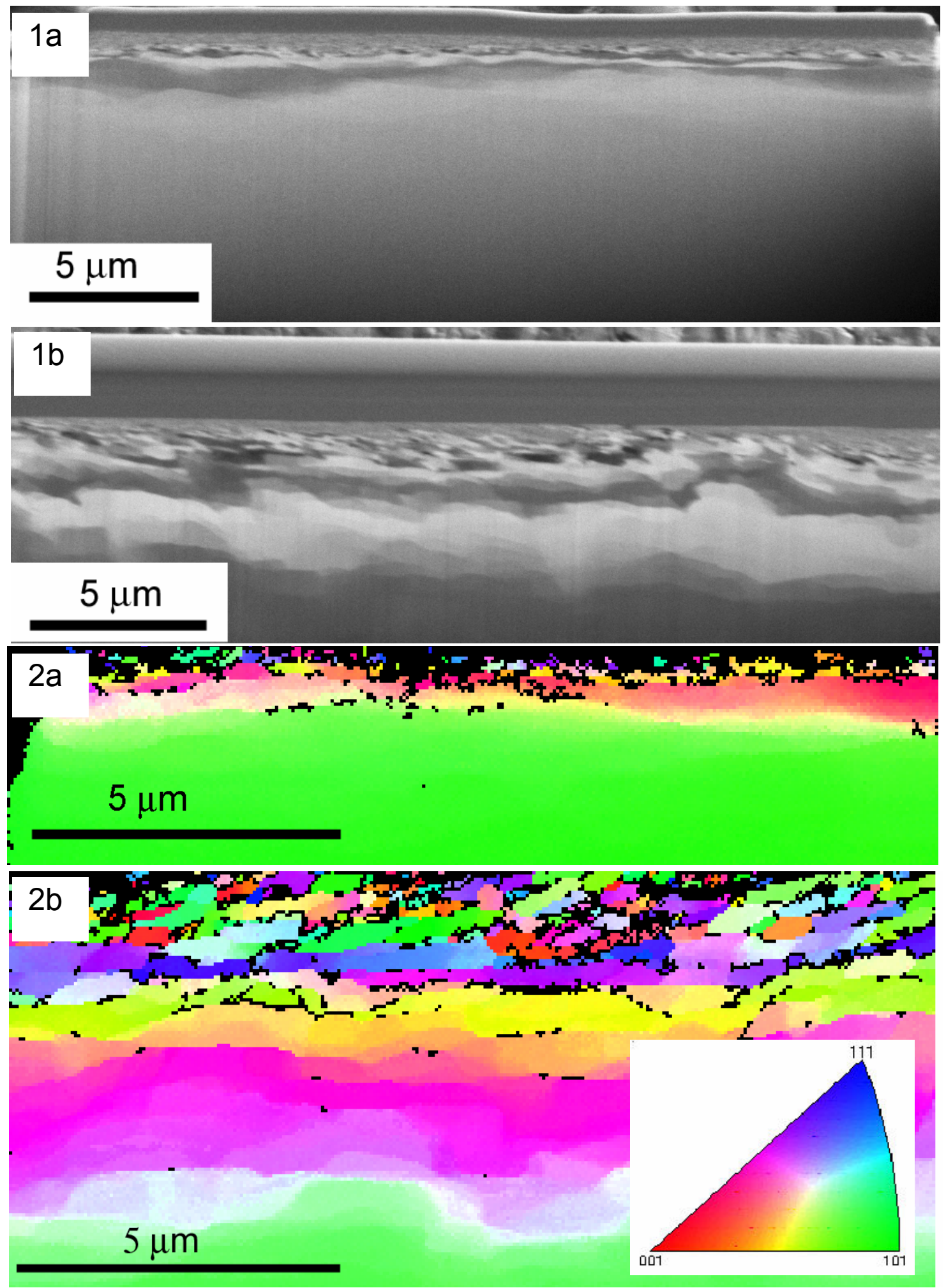

Figure 1. Ion induced secondary electron images of wear-induced deformation on (111) Ni single crystals in the $<110>$ direction a) $10 \mathrm{~g}$ load, b) $100 \mathrm{~g}$ load.

Figure 2. Orientation maps the samples shown in Fig 1 with respect to the sliding direction. a) 100 $\mathrm{mN}$ load, b) $1 \mathrm{~N}$ load. Note the extensive amount of deformation that has occurred. 